\title{
Trust in Personalized Web Search based on Clustered Query Sessions
}

\author{
Suruchi Chawla \\ Assistant Professor \\ Department of Computer Science \\ Shaheed Rajguru College of Applied Science,University of Delhi, Vasundhra Enclave
}

\begin{abstract}
Personalized Web Search customizes the search according to the information need of the user on the Web. In Personalized Web search, search is personalized using the search results of those users profile which have the information need similar to that of present/current user. It is realized that similarity measure of the users profile is not the sufficient criterion for identifying the trusted users profile who are good in generating the effective personalized search results. One of the Research done in Personalized Web Search in [10] in which clustered user query sessions are used for generating the personalized web search results for the current user using the similarity measure alone. However the method proved to be effective but the use of similarity measure alone could not help in determining how good the clustered user profiles are in generating the reliable recommendations for effective personalized web search. In this paper an algorithm is proposed in which trust is introduced in personalized web search based on clustered query session for which trust metric is defined for clustered user sessions which measure the goodness of the clusters of query sessions in making the reliable recommendations for effective personalized web search and furthermore trust is not static but updated dynamically depending on the response of the user to the personalized search results generated by the selected trusted cluster. The proposed algorithm uses both the trust metric and the similarity measure for selecting the trusted cluster which is similar to the information need of the current user for the personalization of the web search effectively. Experiment was conducted on the clustered user query session to test the effectiveness of the proposed trust based personalized web search and results confirms the effectiveness of the proposed approach.
\end{abstract}

\section{Keywords}

Information Retrieval, Search Engines, Trust, Clustering, Information Scent, Personalized Web Search.

\section{INTRODUCTION}

Personalized Web Search personalizes the user web search according to the Information need of the user. Personalized Web Search improves the precision of the search results by reducing the size of the retrieved search results and increases the number of relevant documents retrieved in the search results by customizing the search according to the information need of the user and hence satisfy the information need of the user effectively. Extensive Research has been done in the area of Personalized Web Search in which different approaches are used for customizing the search results according to the need of the user [23][38] [7] [8] [40] [9] [11] [13] [21] [26]
[16] [37] [44] [18].It is found that Personalized Web search has been accomplished through good user profiling and uses the similarity measure for selecting the most similar user profiles who had already searched on the web with the similar intent as that of current user. These selected user profiles have been used for generating the personalized web search results for the current user. It is realized that similarity measure of the users profile should not be the sole criterion for selecting them for personalizing the web search of the current user. It is realized that it is equally important to determine how good the user profile is in making reliable recommendations for the personalization of the web search for a particular information need of the user. The Trust is the measure of the goodness of the user profile in making reliable recommendations for personalizing the search of the current user. Thus both the trust and similarity measure are required to select the trustworthy users profiles who already searched on the web with the similar intent as that of current user in order to generate the reliable recommendations for personalizing the web search of the current users.

In this paper trust metric is defined for clustered user profiles and is used in Personalization of web search based on the clustered user sessions for increasing the effectiveness of personalized web search proposed in [10]. In [10] the clustered user query sessions keyword vector on the web are used to personalize the user search on the web. During online web search, the input query is used to select the clusters using similarity measure. The selected clusters are used to recommend URLs for Personalized Web Search. The current user's clicks to the personalized search results are tracked to infer his partial information need which is further used to select the most similar clusters for the generation of recommendation for the next result page. The process of recommendation goes on till user continues with his current search session.

An algorithm is proposed in this paper for introducing trust in the Personalized Web Search based on clustered query sessions. Further Trust value is not static but it is changed dynamically according to the user feedback on the recommended results in personalized search results which ultimately determines the reliability of the recommendations generated by the selected clustered users profile. Thus the high trust values indicates the high reliability of selected clustered user profiles in predicting the information need of the current user and hence it indicates that most of the time the URLs recommended by the selected user profile in the personalized results are clicked by the current user and viceversa.

Experimental Study was conducted on the data set of user query sessions captured on the web to test the effectiveness of 
the Trust based Personalized Web Search(PWS) using clustered query sessions. The experimental results confirm the effective improvement of precision of personalized search results using the Trust based PWS as compared to PWS without trust based on same clustered query sessions.

\section{RELATED WORK}

It is found that recommender system can be more effective by incorporating trust than traditional collaborative filtering. [33][32][30][24][39]. In [3] model based on the social recommendation Process was proposed. In [4] knowledge stored in the form of ontology was used for trust based recommender system. In [46] the problem of cold start while making recommendation for a new user is dealt with. In Golbeck [19] the potential implications for using trust in user interfaces in the area of online social network is shown. A positive relationship between attitude similarity and friendship has been shown in Burgess and Wallin [5] and Byrne [6]. The comparative study of existing techniques of using trust for effective web search is given below in Table 1 .

Table 1. Comparative Study of Existing Techniques of using Trust for Effective Web Search.

\begin{tabular}{|c|c|c|}
\hline Techniques & Methods & Effectiveness \\
\hline $\begin{array}{l}\text { In [34] trust based } \\
\text { recommender } \\
\text { system is } \\
\text { proposed. }\end{array}$ & $\begin{array}{l}\text { In this a technique } \\
\text { is proposed using } \\
\text { both trust metric } \\
\text { and similarity } \\
\text { metric. }\end{array}$ & $\begin{array}{l}\text { The coverage is } \\
\text { increased, but the } \\
\text { binary relationship } \\
\text { on the basis of } \\
\text { distance are the } \\
\text { limitations. }\end{array}$ \\
\hline $\begin{array}{l}\text { In [48] A new } \\
\text { trust model based } \\
\text { on social } \\
\text { characteristic and } \\
\text { reputation } \\
\text { mechanism for the } \\
\text { semantic web was } \\
\text { proposed. }\end{array}$ & $\begin{array}{l}\text { In this agents were } \\
\text { allowed to decide } \\
\text { which among } \\
\text { different sources } \\
\text { of information to } \\
\text { trust. }\end{array}$ & $\begin{array}{l}\text { The agents act } \\
\text { rationally on the } \\
\text { semantic web } \\
\text { using proposed } \\
\text { techniques. }\end{array}$ \\
\hline $\begin{array}{lr}\text { In [45] } & \text { Trust } \\
\text { model based } & \text { on } \\
\text { reputation } & \text { for } \\
\text { peer-to-peer } & \\
\text { networks } & \text { was } \\
\text { proposed. } & \end{array}$ & $\begin{array}{l}\text { In this the trust } \\
\text { value of a given } \\
\text { peer tras } \\
\text { computed using its } \\
\text { local trust } \\
\text { information and } \\
\text { recommendations } \\
\text { from other nodes }\end{array}$ & $\begin{array}{l}\text { The proposed } \\
\text { model is more } \\
\text { robust on trust } \\
\text { security problems. }\end{array}$ \\
\hline $\begin{array}{lr}\operatorname{In}[25] & \\
\text { TrustWalker: } & \text { A } \\
\text { Random } & \text { Walk } \\
\text { Model } & \text { is } \\
\text { proposed. } & \end{array}$ & $\begin{array}{l}\text { An algorithm is } \\
\text { proposed for } \\
\text { combining trust- } \\
\text { based and item- } \\
\text { based } \\
\text { recommendation. }\end{array}$ & $\begin{array}{ll}\text { The algorithm } & \text { is } \\
\text { limited } & \text { to } \\
\text { centralized } & \\
\text { systems only. } & \end{array}$ \\
\hline $\begin{array}{lr}\text { In } & \text { [27] } \\
\text { Collaborative } & \\
\text { Filtering } & \text { is } \\
\text { improved } & \text { with } \\
\text { Trustbased } & \\
\text { Metrics". } & \end{array}$ & $\begin{array}{l}\text { In this trust metric } \\
\text { is defined for } \\
\text { incorporating trust } \\
\text { in similarity } \\
\text { computation. }\end{array}$ & $\begin{array}{l}\text { A } r \text { trust } \\
\text { propagation graph } \\
\text { based on trust } \\
\text { metric generates } \\
\text { closer to accurate } \\
\text { recommendations. }\end{array}$ \\
\hline $\begin{array}{l}\text { In [31] a Trust- } \\
\text { based } \\
\text { Recommender }\end{array}$ & $\begin{array}{l}\text { The users are } \\
\text { joined by trust } \\
\text { scores connected }\end{array}$ & $\begin{array}{l}\text { The information } \\
\text { stored in trust } \\
\text { network form the }\end{array}$ \\
\hline
\end{tabular}

\begin{tabular}{|c|c|c|}
\hline $\begin{array}{ll}\text { System } & \text { for } \\
\text { Collaborative } & \\
\text { Networks } & \text { is } \\
\text { proposed. } & \end{array}$ & $\begin{array}{ll}\text { through } & \text { trust } \\
\text { network. } & \end{array}$ & $\begin{array}{l}\text { basis of most of } \\
\text { the Trust based } \\
\text { method. [43][49] }\end{array}$ \\
\hline $\begin{array}{l}\text { In [20] Method of } \\
\text { Propagation of } \\
\text { trust and distrust } \\
\text { is introduced. }\end{array}$ & $\begin{array}{l}\text { A framework of } \\
\text { trust propagation } \\
\text { schemes in a large } \\
\text { trust network, is } \\
\text { proposed. }\end{array}$ & $\begin{array}{lr}\text { The trust } & \text { is } \\
\text { predicted } & \text { with } \\
\text { high accuracy } \\
\text { using } & \text { small } \\
\text { number } & \text { of } \\
\text { expressed } & \\
\text { trusts/distrust } & \text { per } \\
\text { individual. } & \end{array}$ \\
\hline $\begin{array}{lr}\text { In } & \text { [50] } \\
\text { Interactions } & \text { of } \\
\text { Trust and Interest } \\
\text { Similarity is } \\
\text { investigated. }\end{array}$ & $\begin{array}{l}\text { It is argued that } \\
\text { for getting } \\
\text { meaningful } \\
\text { results, trust must } \\
\text { reflect user } \\
\text { similarity to some } \\
\text { extent. }\end{array}$ & $\begin{array}{l}\text { A proposed } \\
\text { framework } \\
\text { suggests that there } \\
\text { exists positive co- } \\
\text { relationship } \\
\text { between trust and } \\
\text { interest similarity. }\end{array}$ \\
\hline $\begin{array}{l}\text { In [36] the trust- } \\
\text { based weighting } \\
\text { and trust-based } \\
\text { filtering has } \\
\text { incorporated trust } \\
\text { into collaborative } \\
\text { recommendation } \\
\text { process. }\end{array}$ & $\begin{array}{l}\text { The profile-level } \\
\text { or item-level trust } \\
\text { metrics are } \\
\text { proposed which } \\
\text { has been used } \\
\text { with both trust- } \\
\text { based weighting } \\
\text { and trust-based } \\
\text { filtering. }\end{array}$ & $\begin{array}{l}\text { The prediction } \\
\text { error rate is } \\
\text { reduced } \\
\text { significantly. }\end{array}$ \\
\hline
\end{tabular}

\section{BACKGROUND}

\subsection{Trust}

The concept of Trust has been gaining increase amount of attention in research communities like online recommender system. Trust has been defined and used in many different ways. A trust is defined as social phenomena and the model of trust for artificial world like web is based on how trust works between people in society.[1] Although vast literature on trust has grown in various areas of research with varying meaning of trust but a complete formal unambiguous definition of trust exists rarely in the literature.[35]

One of the definition of trust given by Dasgupta is "the expectation of one person about the actions of others that affects the first persons choice, when an action must be taken before the actions of others are known" [14]. In another definition given by Gambetta [17], it is quoted as "trust is a particular level of the subjective probability with which an agent assesses that another agent or group of agents will perform a particular action, both before he can monitor such action and in a context in which it affects his own action". In Keser [29] trust is stated as "trust as the expectation of other persons goodwill and benign intent, implying that in certain situations those persons will place the interests of others before their own". In [28] two general definition of trust is given, one is called reliability trust also called as evaluation trust and other is decision trust. Evaluation trust can be interpreted as the reliability of something or somebody. It can be defined as the subjective probability by which an individual, A, expects that another individual, B, performs a given action on which its welfare depends. On the other hand, the decision trust captures broader concept of trust. It can be 
defined as the extent to which one party is willing to depend on something or somebody in a given situation with a feeling of relative security, even though negative consequences are possible. In [2] two categories of the trust is defined one is Context-specific interpersonal Trust and second is system / impersonal trust. In Context-specific interpersonal Trust, user trust another user with respect to one specific situation but not necessarily another. In system/impersonal trust, user trust in a system as a whole.

\section{Characteristics}

In [15] the general properties of trust in e-services were surveyed and analyzed and the general properties of trust are listed as follows:

- Trust is relevant to specific transactions only.

- Trust is a measurable belief.

- Trust is directed.

- Trust exists in time.

- Trust evolves in time, even within the same transaction..

- Trust between collectives does not necessarily distribute to trust between their members.

- Trust is reflexive,

- Trust is a subjective belief.

It is found that trust-based recommendations outperformed collaborative filtering algorithms in certain cases.

In [36] the "trust" is defined as the reliability of a partner profile to deliver accurate recommendations in the past. Two models of trust called profile and item level are described for generating reliable and accurate recommendations.

Thus this trust has been incorporated into collaborative recommendation process and hence generates trust-based weighting and trust-based filtering, both of which can be used with either profile-level or item-level trust metrics. It is found that use of trust values has the positive impact on the overall prediction error rate thus significantly reducing the prediction error rate and thereby improves the prediction accuracy. The research work proposed in this paper is inspired from the work proposed in [36].

\subsection{Information Scent}

Information scent is the sense of value and cost of accessing a page based on perceptual cues with respect to the information need of user. Information Scent is measure of degree of relevancy of clicked URLs with respect to the information need of the user. [41][42]

\subsubsection{Information Scent metric}

The Inferring User Need by Information Scent (IUNIS) algorithm is used to quantify the Information Scent $s_{\text {id }}$ of the pages $P_{i d}$ clicked by the user in $i^{\text {th }}$ query session [12][22]. The information scent $s_{\text {id }}$ is calculated for each clicked page $P_{\text {id }}$ in a given query session $\mathrm{i}$ for all $\mathrm{m}$ query sessions identified in query session mining as follows

$\mathrm{Sid}=\mathrm{PF} . \mathrm{IPF}\left(\mathrm{Pid}_{\mathrm{d}}\right) * \mathrm{Time}\left(\mathrm{Pid}_{\mathrm{id}}\right) \forall \mathrm{i} \in 1 . . \mathrm{m} \forall \mathrm{d} \in 1 . . \mathrm{n}$ (1)

$$
\begin{gathered}
P F . I P F\left(P_{i d}\right)=\mathrm{fP}_{\mathrm{id}} / \max \left(\mathrm{fP}_{\mathrm{Pid}}\right) * \log \left(\mathrm{M} / \mathrm{m}_{\mathrm{Pd}}\right) \\
\mathrm{d} \in 1 \ldots \mathrm{n}
\end{gathered}
$$

PF.IPF $\left(\mathrm{P}_{\mathrm{d}}\right)$ : $\mathrm{PF}$ correspond to the page $\mathrm{P}_{\mathrm{id}}$ normalized frequency $f_{P i d}$ in a given query session $i$ where $n$ is the number of distinct clicked page in session $\mathrm{i}$ and IPF correspond to the ratio of total number of query sessions $\mathrm{M}$ in the whole data set to the number of query sessions $m_{P d}$ that contain the given page $P_{d}$.

Time(Pid) given session $i$ to the total duration of query session i.[10]

\subsubsection{Generation of Query sessions keyword vector for Clustering}

Each query session keyword vector is generated from query session containing the clicked URLs where each clicked URLs are those URLs which user clicked in the search results of the input query before submitting another query.

The query session keyword vector $Q_{i}$ of the $i^{\text {th }}$ session is defined as linear combination of TF.IDF content vector of each clicked page $P_{\text {id }}$ scaled by the weight $s_{\text {id }}$ which is the information scent associated with the clicked page $P_{i d}$ in session $i$. That is

$$
\mathrm{Q}_{\mathrm{i}}=\sum_{\mathrm{d}=1}^{\mathrm{n}} \mathrm{Sid}^{*} * \mathrm{Pid}_{\mathrm{id}} \quad \forall \mathrm{i} \in 1 . . \mathrm{m}(3)
$$

where $\mathrm{n}$ is the number of distinct clicked pages in the session i Each $i^{\text {th }}$ query session is obtained as weighted vector $\mathrm{Q}_{\mathrm{i}}$ using formula (3). This vector is modeling the information need associated with the $i^{\text {th }}$ query session.

The k-means algorithm is used for clustering this resulting query sessions keyword vectors and the criterion function is used for measuring the quality of resulting clusters.[47][51][52].

\section{TRUST BASED PERSONALIZED WEB SEARCH}

In this paper trust is used in personalized web search based on clustered query sessions. Trust metric is defined for each clicked URLs of the clustered query sessions using recommended and clicked count of the clicked URLs. The trust metric of the clicked URLs of the particular cluster is further used to derive the trust associated with the cluster.

The processing of the algorithm proposed for Trust Based Personalized Web Search is divided into two phase: Phase I and Phase II. Phase I describes the offline preprocessing and Phase II describes the online processing for the trust based personalized web search.

\section{Phase I}

In Phase I the data set containing the input query and clicked URLs of the users on the web are preprocessed to get query session. The query session keyword vector is generated from query session using Information Scent and content of the clicked URLs. These query session keyword vector are clustered using k-means algorithm. In order to define trust both at clicked URLs of the query sessions and at the cluster level, two values recommended count and clicked count are 
associated with each distinct clicked URLs of the cluster. The recommended count and clicked count records the number of times it is recommended and number of times it is actually clicked by the user when recommended. These counts are initially zero and trust associated with the each cluster is initially undefined in offline processing. The steps involved in offline processing are given below.

\section{Offline Preprocessing}

1. Data Set Collected on the Web is preprocessed to get the Query Sessions.

2. For each clicked URLs, the Information Scent Metric is calculated which is the measure of the relevancy of the clicked URLs with respect to the information need of the user.

3. Query sessions keyword vector is generated from query sessions using Information Scent and content of Clicked URLs.

4. k-means algorithm is used for clustering query sessions keyword vector.

5. Each cluster $\mathrm{i}$ is associated with the mean keyword vector cluster ${ }_{i} \_$mean.

6. Clicked count and recommended count associated with each distinct clicked URLs in the given cluster are initialized to zero.

7. For each distinct clicked URL in the each cluster $\mathrm{i}$ initialize $\operatorname{Trust}\left(\right.$ ClickedURL $\left._{\mathrm{i}}\right)=0$.

8. For each cluster $\mathrm{i}$ the initially the trust is undefined TrustDefined (i)=false and Trust $(i)=0$

\section{Phase II}

During online processing, initially the input query is used to select the cluster which is most similar to the information need of the user. The selected cluster is used to select the high scent clicked URLs for recommendation at the same time the recommended count of each selected clicked URL is increased by one and Trustdefined status of the selected cluster becomes true if false. The clicked count of each recommended URL is increase by one for each click it is received by the user. Thus trust metric is computed for each clicked URL in selected cluster using recommended and clicked count. The trust metric of the clicked URL in the selected cluster is further used to derive the trust value of the selected cluster. Once the trust metric is defined for clusters, then both the trust and similarity measure are used in future to select the clusters for recommendations. The steps involved in online processing are given below.

\section{Online Preprocessing}

1. The input query is used to find the most similar cluster.

2. For each cluster $i$ the similarity is measured using the formulae

MatchScore $_{i}\left(\right.$ input query , cluster $\left._{i}\right)=\{$ 2 (sim(input query,cluste ${ }_{i}$ mean)* Trust (i))/ Trust (i) $+\operatorname{sim}\left(\right.$ cluster $_{i} \_$mean,input query). when TrustDefined(i) $=$ True $\operatorname{sim}($ input query
when TrustDefined(i) $=$ False

3. Identify the most matching cluster i.

4. if for the selected cluster $i$ the TrustDefined(i)=true then

Identify the clicked URLs in selected cluster $\mathrm{i}$ whose Trust value Trust(ClickedURL $\left.\mathrm{i}_{\mathrm{i}}\right)>=\varepsilon$ and store it in list $\mathrm{L}$.

Else

TrustDefined(i)=true
Identify the clicked URLs in selected
cluster i whose Information
Scent $\left(\right.$ ClickedURL $\left.L_{\mathrm{i}}\right)>=\rho$ and store it in
list $\mathrm{L}$.

5. The selected URLs in the list $\mathrm{L}$ is recommended to the user and the Recommendedcount of the corresponding recommended URLs in the selected cluster are incremented by 1 .

6.The user response to the recommended URLs is tracked and stores it in current user profile.

7. For each recommended URL clicked by the user, the clicked count of the corresponding clicked URLs ClickedCount in the selected cluster is incremented by 1 .

8.The trust value of the selected cluster $i$ and clicked URLs present in the selected cluster are calculated as given below.

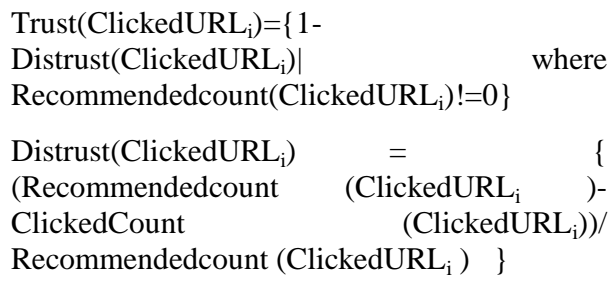

Trust value of the selected cluster $\mathrm{i}$ is defined as follows

Trust (i) $=\{|\operatorname{CorrectSet}(\mathrm{i})| /|\operatorname{RecSet}(\mathrm{i})|\}$

CorrectSet $(\mathrm{i})=\mid\left\{\right.$ ClickedURLs $_{\mathrm{i}} \mid$

Trust $\left(\right.$ ClickedURL $\left._{\mathrm{i}}\right)>\quad \varepsilon \quad$ where

Recommendedcount $\left(\right.$ ClickedURL $\left.\left._{\mathrm{i}}\right) !=0\right\} \mid$

RecSet(i) is the total number of recommendations made using cluster $\mathrm{i}$

$\operatorname{RecSet}(\mathrm{i})=\mid\left\{\right.$ ClickedURLs $_{\mathrm{i}} \mid$

Recommendedcount $\left(\right.$ ClickedURLs $\left.\left._{\mathrm{i}}\right) !=0\right\} \mid$

9. $\quad$ If(Trust $(i)=0)$

TrustDefined $(\mathrm{i})=$ false

10. If the user request for the next result page

a. Model the partial information need of the current user profile using the information scent and content of the URLs clicked so far in his partial user profile and obtain the user session keyword vector current_usersessionvector $\mathrm{r}_{\mathrm{t}}$.

The similarity is measured for each $i^{\text {th }}$ cluster using the formulae 
MatchScore $_{\mathrm{i}}$ (cluster $_{\mathrm{i}}$, current_usersessionvector $\left.r_{t}\right)=2 *(\operatorname{sim}$ (curre nt_usersessionvector, cluster $_{\text {i_ }}$ mean $)^{*}$ Trust (i))/ Trust (i)+sim( cluster $_{i}$ mean, current_usersessionvector ${ }_{t}$ ).when TrustDefined $(\mathrm{i})=$ true

$\operatorname{sim}$ (current_usersessionvector ${ }_{t}$, cluster $_{\mathrm{i}} \mathrm{m}$ ean ) when TrustDefined(i)=false

Goto step 3.

Thus, the trust value associated with the cluster get defined as soon as it is selected for recommendation, furthermore trust value is not static but changes dynamically in response to user feedback on the recommended clicked URLs of the selected cluster. Thus the trust is defined for those clusters whose trust is nonzero and is used with the similarity measure for selecting the clusters for recommendations. This process of recommendation of clicked URLs and updation of trust values continues till the search is personalized to the information need of the current user session.

\section{EXPERIMENTAL STUDY}

Experiment was conducted on the data set of user query sessions collected on the web. The architecture is developed in [10] using JADE, JSP and database Oracle which capture the clicked URLs of users in the search results of Google and performs the personalization of web search without Trust based on clustered query sessions. In order to generate the dataset of user query sessions on the web, the user is required to enter the input query through a GUI based interface of the architecture. This input query is passed on to the Google search engine API, and the search results are retrieved and displayed along with the check boxes on the user interface. The user clicks, on the retrieved search results, are captured through the check boxes displayed on the GUI. The captured clicked URLs of user query sessions are stored in the database.

The experiment was performed on Pentium IV PC with 2 GB RAM under Windows XP using JSP, JADE and Oracle. In the experimental set up for evaluating the performance of proposed approach of Personalized Web Search using Trust, the values of the threshold value of Trust $\varepsilon$ is set to 0.5 and threshold value of information scent $\rho$ is set to 0.3 .

The Agents are developed in JADE to perform the Offline processing associated with proposed approach. The tf.idf vector of the clicked URLs of the query sessions are fetched using the Web Sphinx Crawler and loaded into database using oraloader. The clustering agent developed in the JADE is executed to generate query session keyword vector using Information Scent and clustered them using kmeans algorithm. It performs the initialization of the trust of the clusters and the clicked URLs of the query sessions. Snap Shots of the execution of Clustering Agent is shown in Figure 1 .

During Online processing, the input query is issued to GUI based interface designed each for both Personalized Web Search with/without Trust based on the same clustered query sessions dataset. In Personalized Web Search with Trust, the input query is used to find the trustworthy clusters most similar to the information need of the current user. The resultant set of the high trusted clicked URLs associated with the selected clusters are recommended and displayed in the GUI Interface as shown in Figure 2.
The users clicks to the personalized search results are tracked to capture the user's profile and dynamically update the trust associated with the stored clicked URLs and clusters.

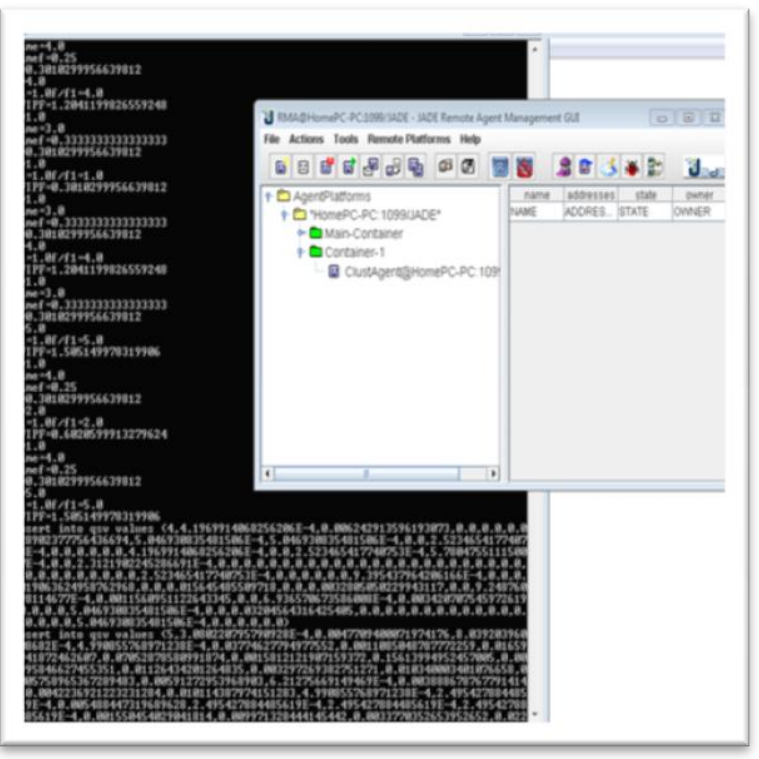

FIG. 1: Screen SnapShot of execution of Clustering Agent.

The performance of the Personalized Web Search using Trust is evaluated from the average precision of personalized search results generated by the proposed approach and compared with Personalized Search Results without Trust proposed in [10]. The Personalized results without Trust are shown in Figure 3 given below.

\begin{tabular}{|l|}
\hline Personalized Search Results with Trust \\
\hline New Hindi Music, Bollywood Songs, Hindi Music New Releases \\
$\square$ http//ww.smashits.com/new-hindi-songs/music-24.html \\
Shanghai (2012) MP3 Movie Songs Download Bollywood Hindi \\
$\nabla$ http://www.djmaza.com/shanghai_2012_mp3_movie_songs_download.html \\
Indian Music, Indian Songs, Hindi Music, Hindi Songs, Music India \\
$\square$ http://ww.smashits.com/music.html \\
Hindi Video Songs, Free Hindi Video Songs, Bollywood Hindi \\
$\square$ http://ww.smashits.com/hindi-songs/movie-videos-list-2.html \\
\hline next
\end{tabular}

\section{FIG. 2: Screen SnapShot of Personalized Search Results with Trust.}

In Personalized Web Search(PWS) with trust the recommended search results are shown in decreasing order of their trust. Highly trusted URLs associated with the selected cluster are listed first. In PWS without Trust there is the case when most trusted URL are listed in the bottom as they are judged on the basis of similarity only. 


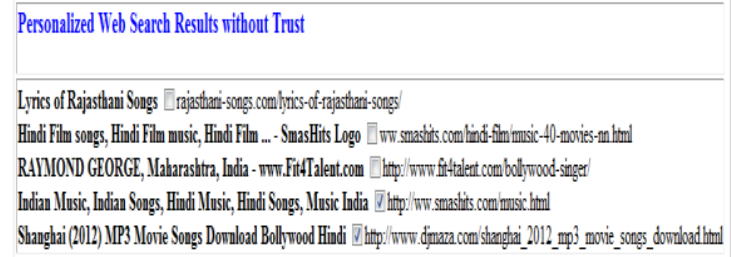

FIG.3: Screen SnapShot of Personalized Search Results without Trust.

In order to evaluate the performance, the test queries were chosen in three domain Academics, Entertainment, Sports. The number of test queries in the three domains was 17 in Academics, 19 in Entertainment and 20 queries in Sports. During online searching, these test queries were issued in each of the selected domain to the GUI based interface to retrieve the personalized search results with/without Trust. The average precision is computed by determining the relevant documents retrieved in the personalized search results.

The experimental results are shown in Figure 4.The obtained results were also analyzed using statistical paired t-test for average precision with Trust and without Trust with 55 degrees of freedom (d.f.) for a combined sample as well as for all three categories (Academics, Entertainment and Sports) with 16 d.f, 18 d.f and 19 d.f. The observed value of $t$ for average precision was 15.70596 for a combined sample. Value of $\mathrm{t}$ for paired difference of average precision was 15.00097 for academics, 8.851583 for entertainment and 20.23376 for sports categories. It was observed that the computed $t$ value for paired difference of average precision lie outside the $95 \%$ confidence interval in each case. Hence Null hypothesis was rejected and alternate hypothesis was accepted in each case and it was concluded that average precision is improved significantly using proposed Personalized Web Search with Trust.

The experimental results show that average precision is improved in each of selected domain using proposed approach of Personalized Web Search using Trust. It is shown that personalized search results with Trust retrieves those clicked URLs which are judged not only on the basis of similarity but also on the basis of trust metric as a result of which the number of high quality recommended clicked URLs which satisfies the user information need to the total number of retrieved recommended clicked URLs are more in comparison to that in personalized web search results without trust as the quality of recommended clicked URL in PWS without trust is judged only on the basis of similarity only. Thus the PWS with Trust shows the improvement in precision as comparison to PWS without trust.

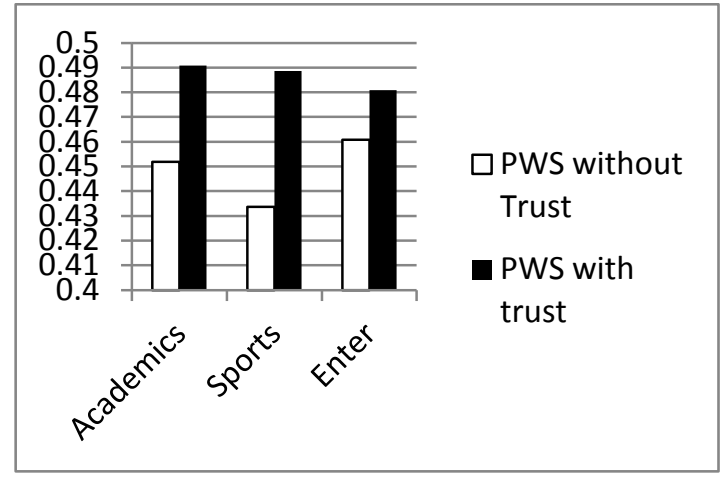

FIG. 4: Shows the avgprecision of Personalized search results with Trust and without Trust.

\section{CONCLUSION}

In this paper trust is introduced in personalized web search based on clustered user query sessions. Trust metric is defined for each cluster and for each clicked URLs in the clusters which ultimately measure how reliable the clusters or the clicked URLs are in satisfying the information need of the user. Initially the trust metric is undefined but it get defined for the clusters and clicked URLs as they get selected for recommendations using the similarity measure only. Once the trust is defined, both the trust and similarity measure are used to select the clusters for recommendations. Furthermore the trust metric of the selected clusters once defined is not static but changes dynamically i.e. increase or decrease with time in response to user clicks to personalized search results. The effectiveness of the proposed approach was evaluated with the experimental study conducted on the user query sessions data set captured in three domain Academics, Entertainment and Sports. The experimental results show the improvement in the precision using proposed trust based personalized web search.

\section{REFERENCES}

[1] Abdul-Rahman, A., \& Hailes, S. 2000. Supporting trust in virtual communities. Proceedings of the 35th Hawaii International Conference on System Sciences, Hawaii, HI.

[2] Alfarez Abdul-Rahman and Stephen Hailes.1997. A distributed trust model. In New Security Paradigms 1997, 48-60.

[3] Bedi, P., \& Kaur, H. 2006. Trust based personalized recommender system. INFOCOM Journal of Computer Science, 5(1), 19-26.

[4] Bedi, P., Kaur, H., \& Marwaha, S.2007. Trust based recommender system for the semantic web, Proceedings of the IJCA/07, Hyderabad, India. 2677-2682.

[5] Burgess, E. and Wallin, P. 1943. Homogamy in Social Charastics. American Journal of Sociology. Vol. 49, No. $2,117-124$

[6] Byrne, D. 1961. Interpersonal Attraction and Attitude Similarity. Journal of Abnormal and Social Psychology. 62(3), 713-715

[7] Burke, R. 2007. Hybrid Web Recommender Systems. The Adaptive Web, 377-408.

[8] Burke, R. 2002. Hybrid Recommender Systems: Survey and Experiments. 
[9] Chan, P.K. 2000. Constructing Web User Profiles: A non-invasive Learning Approach. Lecture Notes In Computer Science, Volume 1836, 39-55.

[10] Chawla Suruchi and Bedi Punam.2007. 'Personalized Web Search using Information Scent' in CISSE'07 :Proceedings of the International Joint Conferences on Computer, Information and Systems Sciences, and Engineering, Technically Co-Sponsored by: Institute of Electrical \& Electronics Engineers (IEEE), University of Bridgeport, published in LNCS (Springer), December 312.

[11] Chen, L., Pu, P. 2004. Survey of Preference Elicitation Methods. Technical Report IC/200467, Swiss Federal Institute of Technology in Lausanne.

[12] Chi, E H., Pirolli, P., Chen K. and Pitkow J. 2001. 'Using Information Scent to model User Information Needs and Actions on the Web' in ACM CHI 2001: Proceedings of the Conference on Human Factors in Computing Systems, New York,NY, USA, 490-497.

[13] Chirita, P.A., Nejdl, W., Paiu, R., Kohlschtter, C. 2005. Using ODP Metadata to Personalize Search. Proceedings of the 28th annual international ACM SIGIR conference on Research and development in information retrieval, 178-185.

[14] Dasgupta, P. 1990. Trust as a Commodity. In D. Gambetta (Ed.), Trust: Making and Breaking Cooperative Relations. Oxford: Basil Blackwell.

[15] Dimitrakos, T. 2003. A Service-Oriented Trust Management Framework. International Workshop on Deception, Fraud \& Trust in Agent Societies, 53-72.

[16] Ferragina, P., Gulli, A. 2005. A Personalized Search Engine Based on WebSnippet Hierarchical Clustering. Special interest tracks and posters of the $14^{\text {th }}$ International WWW Conference, 801-810.

[17] Gambetta, D. (Ed.). 2000. Can We Trust Trust? (Vol. 13). Oxford: University of Oxford

[18] Gauch, S., Chaffee, J., Pretschner, A. 2003. OntologyBased User Profiles for Search and Browsing. Web Intelligence and Agent Systems, Volume 1, 219-234.

[19] Golbeck, J. 2009. Trust and Nuance Profile Similarity in Online Social Network. ACM Transactions on the Web. 3(4), 12.1-33.

[20] Guha, R. V., Kumar, R., Raghavan, P., Tomkins, A., 2004. Propagation of trust and distrust. The Proceedings of the 13th International World Wide Web Conference,USA, 403-412.

[21] Haveliwala., T. 2002. Topic-sensitive pagerank. Proceedings of the 11th International WWW Conference, 517-526.

[22] Heer, J. and Chi, E.H. 2002.' Separating the Swarm: Categorization method for User Access Session on the Web' in ACM CHI 2002: Proceedings of Conference on Human Factor in Computing System, 243-250.

[23] Hu, J., Zeng, H.-J., Li, H., Niu, C., Chen, Z. 2007. Demographic prediction based on user's browsing behavior. Proceedings of the 16th international conference on World Wide Web, 151-160.
[24] Hwang, C., \& Chen, Y. 2007. Using trust in collaborative filtering recommendation. Lecture Notes in Computer Science, Volume 4570, 1052-1060. Innovation Network (2009).

[25] Jamali, M. and Ester M. 2009. TrustWalker: A Random Walk Model for Combining Trust-based and Item-based Recommendation. . Proceedings of the 151th ACM Conference on Knowledge Discovery and Data mining. KDD.09, Paris, France.

[26] Jeh, G., Widom, J. 2003. Scaling personalized web search. Proceedings of the $12^{\text {th }}$ International WWW Conference, 271-279.

[27] Jianshu Weng, Chunyan Miao, Angela Goh.2006 "Improving Collaborative Filtering with Trustbased Metrics”, SAC'06, April, 2327, Dijon, France, ACM 1595931082/ 06/0004

[28] Jøsang, A. 2007. Probabilistic Logic Under Uncertainty. In The Proceedings of Computing: The Australian Theory Symposium (CATS2007), CRPIT Volume 65, Ballarat, Australia.

[29] Keser, C. 2003. Experimental games for the design of reputation management systems. IBM Systems Journal, 42(3), 498-506.

[30] Lathia, N., Hailes, S \& Capra, L. 2008. Trust-based collaborative filtering. Proceedings of the joint iTrust and PST Conference on Privecy, Trust Management and Security. Springer, 119-134 .

[31] Leonardo Zanette, Claudia L.R. Motta, Flávia Maria Santoro, Marcos Elia. 2009. "A Trust-based Recommender System for Collaborative Networks", IEEE.

[32] Levien, R. 2004. Attack-resistant Trust Metrics. Ph.D. thesis, University of California at Berkeley, USA.

[33] Massa, P., \& Avesani, P. 2007. Trust-aware Recommender Systems. Proceedings of the ACM Conference on Recommender Systems, 17-24.

[34] Massa, P., \& Bhattacharjee, B. 2004. Using trust in recommender systems: An experimental analysis. Proceedings of the Second International Conference on Trust Management, Oxford, UK., 221-235.

[35] McKnight, D. H., \& Chervany, N. L. 2002. What Trust Means in e-Commerce Customer Relationships: An interdisciplinary conceptual typology. International Journal of Electronic Commerce, 6(2), 35-59.

[36] O'Donovan, J. and Smyth, B.2005. Trust in Recommender Systems. Proceedings of the $10^{\text {th }}$ International Conference on Intelligent User Interfaces. 167-174.

[37] Pahlevi, S.M., Kitagawa H. 2002. Taxonomy-based Adaptive Web Search Method. 3rd IEEE International Conference on Information Technology: Coding and Computing.

[38] Pazzani, M.J.1999. A Framework for Collaborative, Content-Based and Demographic Filtering.

[39] Peng, T., \& Seng-cho, T. 2009. iTrustU: A blog recommender system based on multifaceted trust and collaborative filtering. Proceedings of the ACM 
Symposium on Applied Computing. New York, NY. 1278-1285.

[40] Pierrakos, D., Paliouras, G., Papatheodorou, C., Spyropoulos, C.D. 2003.Web Usage Mining as a Tool for Personalization: A Survey. User Modeling and UserAdapted Interaction, 13(4), 311-372.

[41] Pirolli, P. 1997. 'Computational models of information scent-following in a very large browsable text collection' in ACM CHI 97: Proceedings of the Conference on Human Factors in Computing Systems, 3-10.

[42] Pirolli, P. 2004.The use of proximal information scent to forage for distal content on the world wide web. In Working with Technology in Mind: Brunswikian. Resources for Cognitive Science and Engineering, Oxford University Press.

[43] Rashmi Sinha and Kirsten Swearingen.2001. Comparing Recommendation made by Online Systems and Friends. In the Proceedings. of the DELOS-NSF Workshop on Personalization and Recommender Systems in Digital Libraries, Dublin,Ireland.

[44] Sugiyama, K., Hatano, K., Yoshikawa, M. 2004. Adaptive Web Search Based on User Profile Constructed without Any Effort from Users. Proceedings of the $13^{\text {th }}$ international WWW Conference, 675-684.

[45] Tian, C.-Q., Zou, S.-H., Wang, W.-D., \& Cheng, S.D.2008. Trust model based on reputation for peer-to-peer networks. Journal on Communication, 29(4), 63-70.
[46] Victor, P., De Cock, M., Camelis, C., \& Teredesai, A.2008. Getting cold start users connected in a recommender systems trust network. Computational Intelligence in Decision and Control, Volume 1. 877 882 .

[47] Wen, R J., Nie, Y J. and Zhang, J H.2002. Query Clustering Using User Logs. ACM Transactions on Information Systems, 20(1),59-81.

[48] Xue, W., \& Fan, Z. 2008. A new trust model based on social characteristic and reputation mechanism for the semantic web. Proceedings of the Workshop on Knowledge Discovery and Data Mining.

[49] Zhili Wu, Xueli Yu and Jingyu.2009. An Improved Trust Metric for Trust-aware Recommender Systems. IEEE.

[50] Ziegler, C. N. and Golbeck, J. 2007. Investigating Interactions of Trust and Interest Similarity. Decision Support Systems. Volume. 43, 460-475.

[51] Zhao, Y. and Karypis, G.2002a. Comparison of agglomerative and partitional document clustering algorithms. In SIAM Workshop on Clustering Highdimensional Data and its Applications.

[52] Zhao, Y. and Karypis, Y. 2002b. Criterion functions for document clustering. Technical report, University of Minnesota, Minneapolis, MN. 